\title{
Design and Accuracy Evaluation of High-Speed and High Precision Parallel Mechanism
}

\author{
Yoshihiko KOSEKI*, Tatsuo ARAI**, Kouichi SUGIMOTO*** \\ Toshiyuki TAKATUJI****, Mitsuo GOTO**** \\ *Mechanical Engineering Laboratory, AIST, \\ Namiki1-2, Tsukuba, Ibaraki 305, Japan, e-mail to koseki@mel.go.jp \\ **Graduate School of Engineering Science, Osaka University, Japan \\ ***Hitachi ltd., Japan, ****National Research Laboratory of Metrology, AIST, Japan
}

\begin{abstract}
We have developed a precise and high-speed arm applied to assembly processes in electrical product and machine industries. The arm is designed based on the requirement from Japanese major industries. The paper addresses the background of the development, the required specification for the robot arm, and the brief introduction of the prototype arm and evaluations of accuracy. We test the repeatability of parallel mechanism. Then we calibrate the kinematic model of parallel mechanism to improve its absolute accuracy using laser tracking coordinate measuring system.

Key words: parallel mechanism, accuracy, calibration, high speed and assembly
\end{abstract}

\section{Introduction}

Robot manipulators have been contributing to the advancement of automation for machining and assembling processes but a lot of tasks are still left for manual works, especially assembly.

For promotion of automation, we survey and discuss the problem of conventional industrial robot and the requirement for future industrial robot. The results are mentioned below and we can point out that the fatal problems in general application is programming. In addition, it is necessary for complicated assembly task to develop high speed of force control.

The conventional programming and playback is essential to industrial robot and spends much time and cost. Robotic intelligence is ultimate solution, but difficult. The off-line programming method is another possible solution. If robot were ideally accurate and modeled, all the motion of robot could be decided in computer. It can be concluded that absolute positioning accuracy is essential to off-line programming.

Parallel mechanisms have many excellent characteristics compared to a serial mechanisms widely applied to conventional industrial robots. Many researches and developments have been done, i.e., high speed arms [1][2][3], a high power arm [4] and micro mechanisms [5][6] have been proposed. However, the parallel mechanism has not widely been utilized as a commercial industrial robot.

The paper discusses the results of the survey, the specification and the design of a new arm based on the parallel mechanism, and its basic characteristics with evaluation experiments. We experimentally evaluate the accuracy of parallel mechanism using laser tracking coordinate measuring system. This system has many characteristics that suit for robot calibration and we would introduce this system, calibration strategy, its result and discussions. We also apply the Adept motion to evaluate its high-speed capability.

\section{Design of prototype arm}

\section{1 Questionnaire on the Current Industrial Robots}

For the Japanese major companies of electrical appliance, electronics, and heavy industries, which are user and/or maker of industrial robots, a questionnaire on the problems with and on the requirements for the current industrial robots was made 3 years ago. The questionnaire concluded briefly in the following;

(1) high-speed motion in force control as well as trajectory control,

(2) absolute positioning accuracy,

(3) simple and economical programming,

(4) simple application in assembly tasks, and

(5) more intelligence applicable to complicated tasks.

More detailed analysis in these problems leads that the companies require more excellent characteristics and functions for robot hardware itself. (1), (2) and (3) show the requirements for hardware that can attain high speed and good accuracy. The current industrial robot is based on a serial mechanism configuration that is capable of dexterous motion, although its speed and accuracy are rather limited. If a more excellent mechanism, in terms of 
IEEE International Conference on Robotics and Automation (ICRA), Vol. 3, pp. 1340-1345, 1998

speed and accuracy, is applicable to robot arm, these problems, (1) and (2) may be solved successfully. Furthermore, the requirement (3) may be satisfied, since good positioning accuracy enables an operator to apply an off-line programming method based on design drawings and CAD data. This may reduce the difficulty and the cost in the current programming process and may bring economical contributions.

The requirement (4) indicates that the current force control method applied to assembly does not satisfy the demands of the robot users. One reason might be that the current force control limits the robot motion and results in slow assembly. If the robot hardware has high speed and good accuracy, the force control motion may be improved by using a force sensor and other sensors, and consequently the assembly task may be robotised efficiently. Furthermore, the force control may improve the adaptability of the robot to its environment, and then the robot may obtain more intelligence and attain more complicated tasks.

Based on the above considerations, our project aims to develop a new manipulator that has high speed in motion and force control as well as good absolute positioning accuracy. This robot may contribute to solve the problems and to satisfy the requirements suggested in the questionnaire and may obtain broader application fields. The considered application fields may be the followings in terms of high-speed force control and of good positioning accuracy.

(a) high-speed force control: parts assembly for electric and machine products, debarring, cast finishing, polishing and grinding,

(b) good positioning accuracy: picking and placing, material handling, packing, bonding for ICs, accurate positioning, fine welding, inspection

The robot can be applied in all kinds of conventional positioning tasks. It should be pointed out that even applied to these tasks the programming becomes simpler and less time consuming.

\section{2 Design \& Specification of Prototype}

Based on another survey result on the requirement for assembly robots as well as the discussion mentioned in the previous subsection, the target specification was given for a developed arm in our project, where the application is limited to the assembly for small sized motor, for example. The specifications for mechanism and motion controller are listed in Table 1.

Parallel mechanisms has been expected as a candidate for a novel robot arm and it has been proved by many researchers that it has excellent positioning accuracy and its stiffness, light movable mass, good dynamic characteristic and high force capability are advantageous for force control. Because these merit meet well our specification, parallel mechanism is selected.

Table 1 Specification for the developed arm

\begin{tabular}{|l|l|}
\hline \multicolumn{2}{|c|}{ Mechanism } \\
\hline degrees of freedom & 6 \\
\hline nominal motion range & $300 \times 300 \times 300\left[\mathrm{~mm}^{3}\right]$ \\
\hline pay load & $1 \mathrm{~kg}$ \\
\hline $\begin{array}{l}\text { maximum acceleration } \\
\text { with pay load }\end{array}$ & $10[\mathrm{G}]$ \\
\hline absolute positioning accuracy & $\begin{array}{l}1 / 1000 \text { of the nominal motion range } \\
\text { and compatible to a chamfer }\end{array}$ \\
\hline \multicolumn{2}{|c|}{ Controller } \\
\hline control algorithm & $\begin{array}{l}\text { trajectory and velocity control with } \\
\text { dynamic compensation } \\
\text { high speed force control }\end{array}$ \\
\hline dynamic range in force control & $10[\mathrm{~Hz}]$ at $0.5[\mathrm{~kg}]$ load \\
\hline
\end{tabular}

There are three basic types of parallel mechanisms applicable for robot arm as mentioned, Stewart platform type, a rotary actuation type, a fixed linear actuation type [7], and their modifications. Table 2 shows the comparison of these three mechanisms in terms of basic characteristics including speed, rigidity, workspace, etc. The rotary actuation type is the best in terms of simple actuation mechanism as well as speed, workspace. It looks attractive in our objectives, since it may have less number of mechanical parts and this may lead to less cost in manufacturing.

Table 2 Comparison in three types

\begin{tabular}{|l|l|l|l|}
\hline & rotary & $\begin{array}{c}\text { Stewert } \\
\text { type }\end{array}$ & \\
\hline output force & small & large & large \\
\hline actuator location & basement & moving part & basement \\
\hline moving mass & small & large & small \\
\hline speed & very fast & - & fast \\
\hline rigidity & poor & good & good \\
\hline workspace & large & small & large \\
\hline structure & simple \& & $\begin{array}{l}\text { large moving } \\
\text { parts }\end{array}$ & $\begin{array}{c}\text { large } \\
\text { basemen }\end{array}$ \\
\hline
\end{tabular}

Taking these specifications and characteristics into consideration, we decide on hexa type for the prototype.

\section{3 Prototype overviews}

Based on the design described in the previous section, the prototype arm was built. Fig.1 shows the whole view of the arm.

The end plate and base plate are connected with 6 chains, which consist of rotary actuator and two links that are, input link and coupler link. The input link and the coupler link are jointed by 3 passive joints whose axes 
IEEE International Conference on Robotics and Automation (ICRA), Vol. 3, pp. 1340-1345, 1998

intersect at one point. Those joints have equal function to a spherical joint but larger range of motion. The coupler link and the endplate are jointed by a universal joint.

A 6-axes-force-torque sensor is attached to the endplate for force control and another force-torque sensor is used for joystick control.

Forward and inverse kinematics, position servo, trajectory control, inverse dynamics based on the augmented Lagrange method [8], joystick control [9], force control and Adept motion are implemented to the controller.

Adept motion is reciprocating motion whose stroke and height are $300[\mathrm{~mm}]$ and $25[\mathrm{~mm}]$ respectively and is frequently utilized to estimate the rapidness of robot. We would like to report that we have achieved $0.5[\mathrm{sec}$.] cycle time for the Adept motion with a $2.2[\mathrm{~kg}]$ load mass on the end plate at $300 \%$ rating motor torque. In this case maximum speed is estimated $2.0[\mathrm{~m} / \mathrm{s}]$.

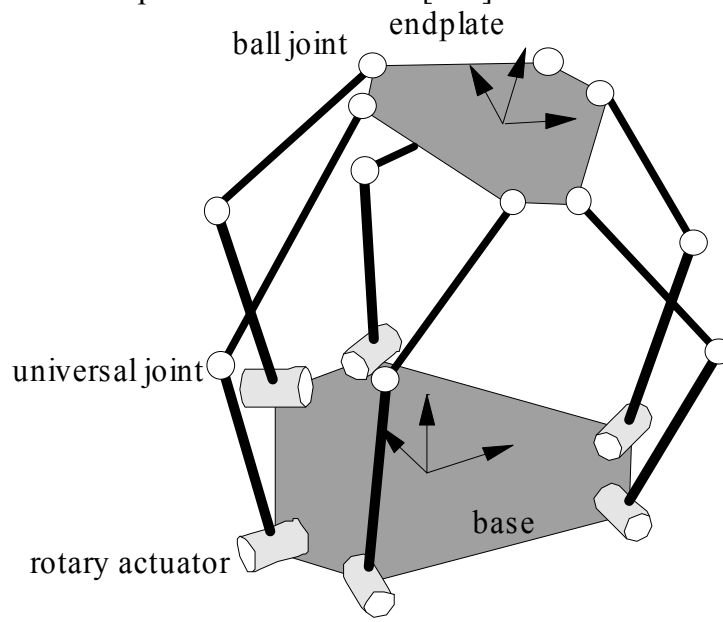

Fig. 1 Overview of the developed arm

\section{Calibration approach and System}

\section{1. Calibration Approach}

The motivation of this project is to develop a practically useful robot for manipulator. The robot calibration is the key to off-line programming, which saves cost of programming.

Calibration needs accurate measurement of output for reference. Conventional coordinate measurement devices do not meet the requirements of calibration, since some are inaccurate and the others have narrow range of measurement.

Some researchers have proposed calibration methods where an end-effector is constrained or angles or positions of intermediate joints or links are measured $[10][11][12]$. The problem of these calibrations is that users can not know how accurate the robot becomes after calibration.

For accuracy evaluation of our parallel mechanism, we would propose the calibration method using laser tracking coordinate measuring system [13][14]. In the following sections, we would introduce the system, the strategy of experiment and its results and discussions.

\section{2. Laser Tracking Coordinate Measuring System}

The laser tracking coordinate measuring system consists of 4 laser stations and a wide-angle retro-reflector, "Cat's Eye". When the laser beam is incident upon the cat's eye, reflected beam returns parallel to the incident beam. The structure of cat's eye is as follows. The cat's eye has a lens whose focus coincides with its spherical surface and the surface mirrors incident beam.

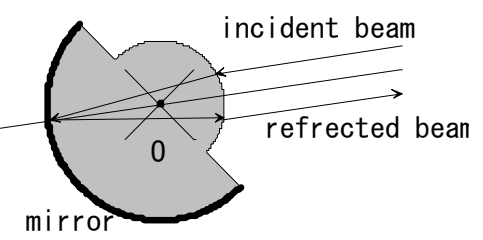

Fig. 2 Retro-reflector, Cat's Eye

The laser station can pan and tilt a laser interferometer and track the target (cat's eye) and measure the distance. This station is controlled keeping the distance between incident beam and reflected beam to zero so that the laser always passes through the center of cat's eye. The interferometer can measure the change in distance from the intersection of two axes of pan and tilt to the cat's eye incrementally. Initial distance, however is not measured. An overview of the laser tracking coordinate measuring system is shown in Fig. 3.

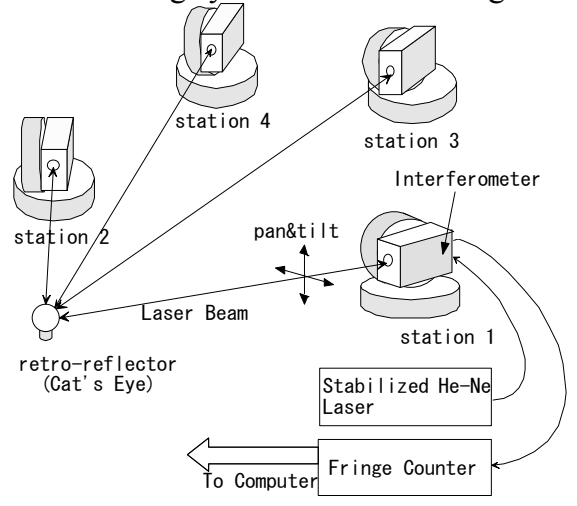

Fig. 3 Laser Tracking Coordinate Measuring System

The target's position was determined if three stations' positions and distances between cat's eye and station were known. This system has $4^{\text {th }}$ additional station to identify stations' positions and initial distances. The distance to the position guessed with inaccurate 
IEEE International Conference on Robotics and Automation (ICRA), Vol. 3, pp. 1340-1345, 1998

parameters can be checked with measured distance. This measurement consists of two processes, redundant measurement and self-calibration. In calibration process, the parameters are modified to minimize the error by the least square method. The calibration requires considerable measurement data.

This system is characterized by following merits and demerits.

(1) Precise measurement with laser interferometer

The other possible methods for wide range coordinate measurement are camera image, PSD (position sensing device), magnetic sensor or ultrasonic sensor. These sensors are, however, can not measure the order of 1 micrometer. There is another measuring system, which uses laser interferometer and senses the direction of laser. This system is not proper to wide range measurement because angle sensor is not precise and the more distant the target is, the larger the measurement error become.

In addition, our measuring system has following merits comparing to the so-called CMM (Coordinate Measuring Machine).

(2) Non-contact measurement

(3) Wide measuring range

(4) Enable to measure a high speed object

These are indispensable for calibration of manipulator, which moves in wide range on high-speed. And it is also a merit that self-calibration with redundant measurement does not need precise setup.

On the contrary, we must take the following demerit into account.

Laser beam must not be interrupted during one set of measurement, because the laser interferometer counts the interference fringe and tracks the target by reflected beam. This restriction is practically important problem because the range of cat's eye is narrower than possible orientation of the endplate and because stations and links of manipulator may interrupt laser beams on some positions and orientations.

\section{Experiment and Accuracy Evaluation}

\section{1. Repeatability test}

Before calibration, we pre-test the repeatability of our parallel mechanism with laser tracking coordinate measuring System. This pretest is partly based on ISO9283.

The manipulator moves to 5 points in $80 \%$ of workspace (See Fig. 4) and at each point the endplate is kept horizontal. The manipulator returns to each point 30 times and moves to 100 random points for calibration of measuring system. The manipulator approaches to each point on single path and random path. In case of single path, it moves from (1) to (5) in order and repeats them.
In case of random path, it approaches to each measure point at random. ISO9283 requires only single path test but repeatability of both paths is also important for actual production line.

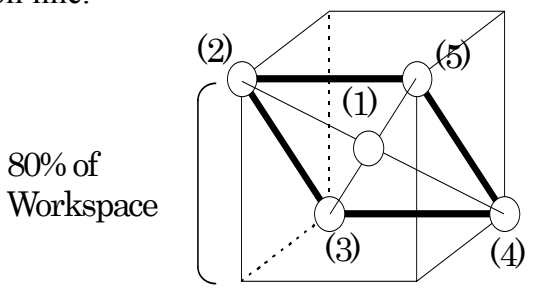

Fig. 4 Measured points of repeatability test

ISO9283 requires the tested manipulator to move at rated speed but in this case the measuring system can not follow the manipulator. Therefore it runs at the maximum $200 \mathrm{~mm} / \mathrm{s}$ with constant acceleration/deceleration.

The results of the repeatability test are shown in Table 3. The error of each point is evaluated with the distance between the center of measured points and each point.

Table 3 Result of Repeatability test

\begin{tabular}{|l|l|l|}
\hline & Max. Error & Ave. Error \\
\hline Single Path & $0.029[\mathrm{~mm}]$ & $0.010[\mathrm{~mm}]$ \\
\hline Random Path & $0.099[\mathrm{~mm}]$ & $0.042[\mathrm{~mm}]$ \\
\hline
\end{tabular}

Table 4 shows the servo error on actuated joint and its contribution to error in the actual workspace. According to these results, in case of single path servo error is dominant and in case of random path, backlash of gears and joints cause error.

Table 4 Error in joint angle and its effect on positioning

\begin{tabular}{|l|l|l|l|l|}
\hline \multirow{2}{*}{} & \multicolumn{2}{|l|}{ Error in Joint Space } & \multicolumn{2}{l|}{ Error in Actual Space } \\
\cline { 2 - 5 } & Max. & Ave. & Max. & Ave. \\
\hline Single & $5.4 \mathrm{e}-5$ & $0.8 \mathrm{e}-5$ & 0.023 & 0.004 \\
Path & {$[\mathrm{rad}]$.} & {$[\mathrm{rad}]$.} & {$[\mathrm{mm}]$} & {$[\mathrm{mm}]$} \\
\hline Random & $4.2 \mathrm{e}-5$ & $1.0 \mathrm{e}-5$ & 0.036 & 0.007 \\
Path & {$[\mathrm{rad}]$.} & {$[\mathrm{rad}]$.} & {$[\mathrm{mm}]$} & {$[\mathrm{mm}]$} \\
\hline
\end{tabular}

\section{2. Absolute accuracy test}

This measuring system can measure the center position of cat's eye; therefore 3 points on the endplate are measured to know the position and orientation of the endplate. 200 test positions are randomly selected in twice extent of the rated workspace, within joint limits and far from singular points. 100 of the 200 points are selected for calibration and the rest is for error evaluation. The robot runs 200 points with cat's eye attached on the endplate and the interferometic distances and angles of input axes are recorded at each point. This experiment is repeated for two other positions of the cat's eye on the endplate. During these experiment four laser stations are fixed in the same places. 
IEEE International Conference on Robotics and Automation (ICRA), Vol. 3, pp. 1340-1345, 1998

The error of measurement depends on accuracy of measuring system and repeatability of manipulator. Usually repeatability error is negligible compared to absolute accuracy. For error evaluation, Fig. 5 shows the distances from one cat's eye to others. We utilize the redundant measurement ( 9 measurements for $6 \mathrm{DOF}$ ) to estimate the measurement error. If error of the measurement would be zero, the distances were constant through the experiment.

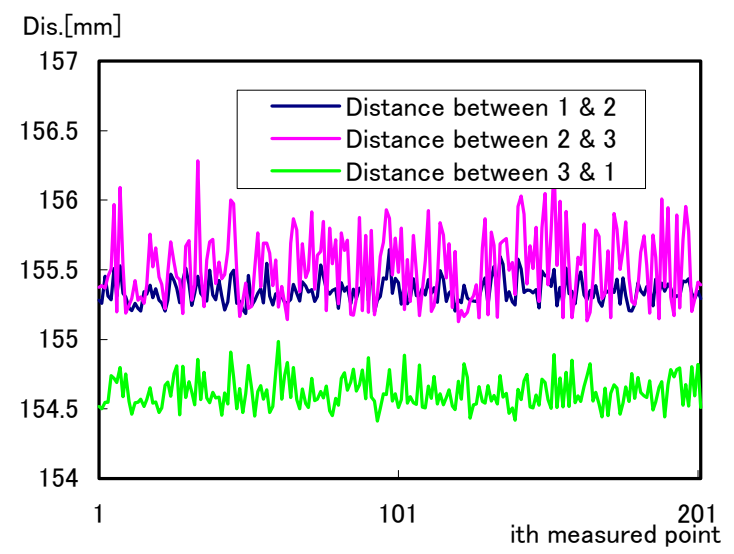

Fig. 5 Distance between cat's eyes

The distance between $2^{\text {nd }}$ and $3^{\text {rd }}$ position varies more than the others and its variance is $0.17 \mathrm{~mm}$ and maximum error is $0.76 \mathrm{~mm}$. This result shows that measuring error is larger than we expected.

To optimize the kinematic parameters, the conventional Newton-Raphson method is adopted. 66 parameters are modified to minimize the sum of squares of error. The error is defined as difference between measured position and orientation of endplate and their predictions by forward kinematics. Each chain of links has 11 parameters, 6 for position and orientation of the input motor, 2 for lengths of the input link and the coupler link, 3 for the position of the coupler link end on the endplate. The manipulator has 6 chains.

Fig. 6, Fig. 7 and Table 5 show the parameters before and after calibration. Fig. 6 and 7 are the top views of the base plate and the endplate, respectively. Table 5 shows calibrated and designed lengths of the input link and the coupler link.

The largest change of parameters is about $10 \mathrm{~mm}$ in position of motor. The noise of measure causes larger change on such parameters.

\section{3. Evaluation of Absolute Accuracy and Discussion}

We evaluate the accuracy of calibration with remaining 100 measurement data. The accuracy is evaluated by distance between commanded position and measured position of the endplate. Table 6 shows the results.

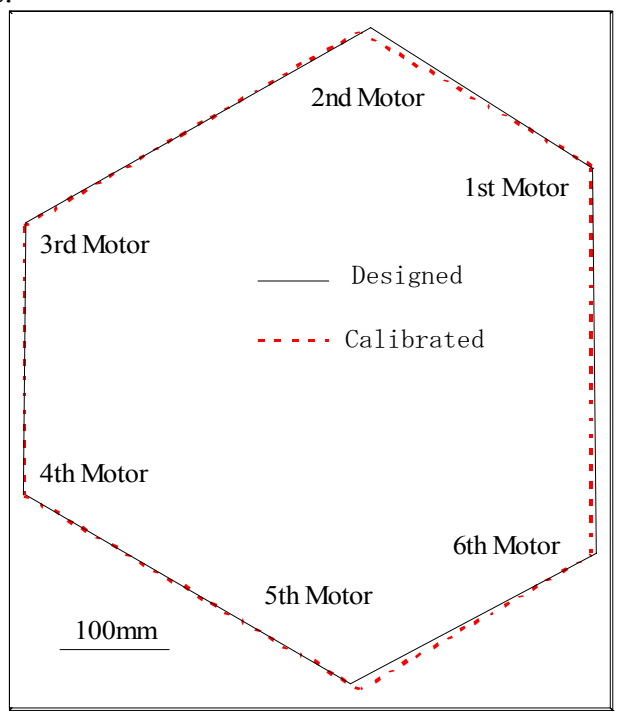

Fig. 6 Calibrated Parameters

- Positions of Motors -

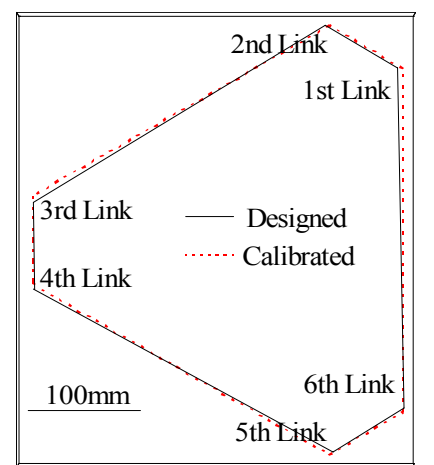

Fig. 7 Calibrated Parameters

- Ends of Links on the Endplate -

Table 5 Calibrated Parameters (Lengths of Links)

\begin{tabular}{|l|r|r|}
\hline & Input Link[mm $]$ & Coupler Link[mm $]$ \\
\hline $1^{\text {st }}$ Links & 703.87 & 939.43 \\
$2^{\text {nd }}$ Links & 705.33 & 936.72 \\
$3^{\text {rd }}$ Links & 704.64 & 940.64 \\
$4^{\text {th }}$ Links & 705.95 & 938.96 \\
$5^{\text {th }}$ Links & 705.37 & 941.33 \\
$6^{\text {th }}$ Links & 705.56 & 942.23 \\
Designed & 705.00 & 940.00 \\
\hline
\end{tabular}

Table 6 Absolute Accuracy

\begin{tabular}{|l|l|l|}
\hline & Max. Error & Ave. Error \\
\hline Before Calibration & $4.68[\mathrm{~mm}]$ & $1.63[\mathrm{~mm}]$ \\
\hline After Calibration & $0.98[\mathrm{~mm}]$ & $0.30[\mathrm{~mm}]$ \\
\hline
\end{tabular}

The absolute error is caused by calibration error, deformation and backlash. Compared with absolute error, 
IEEE International Conference on Robotics and Automation (ICRA), Vol. 3, pp. 1340-1345, 1998

the measurement error is dominant in calibration error. The absolute error and measurement error are $0.98 \mathrm{~mm}$ and $0.76 \mathrm{~mm}$ respectively at the maximum. As mentioned above, the causes of the measurement error can be divided into two groups, measuring system error and the manipulator's repeatability.

According to the pretest, the repeatability of the manipulator influences a little because it is small compared to absolute error, since it's $0.029 \mathrm{~mm}$ on single path. Another cause is stiffness of the manipulator. The cat's eye, which weighs $1100 \mathrm{~g}$, is differently attached at each time and inclines the endplate in different directions within the limits of backlash.

The error of the measuring system is due to the noise on interferometer, servo error, calibration error and the product error of pan \& tilt mechanism and cat's eye. Tiny dust in the air, flaw of cat's eye, electric noise and so on may cause miscounting. The condition is severe for such a long experiment.

The contribution of noises to the result of measurement is indirect and much depends on arrangement of the stations and the manipulator. According to the prior experiments and discussions, this calibration system has singular points and near these points calibration is impossible or the error is increased. The survey of these singular points and their effects are still being studied and developed.

Nakamura reported the calibration of a serial manipulator and its absolute accuracy is $1.8 \mathrm{~mm}[15]$. Though It is difficult to compare both results because mechanism and other specifications are different, it can be concluded that our calibration with the laser tracking coordinate measuring system results in good absolute accuracy.

\section{Conclusions}

We have proposed a parallel arm which is aimed to be used commercially in the industries. We discussed the results of the questionnaire done for the Japanese major companies of maker and user of industrial robots, and showed their requirements clearly. The developed arm was designed by taking account of the requirements and the prototype was built based on the rotary input type parallel mechanism. Then we calibrated the kinematics of the prototype to improve the absolute accuracy, and evaluated the accuracy using laser tracking coordinate measuring System.

\section{Acknowledgements}

The authors would like to express their acknowledgment for IROFA, KHI, Nitta, Hitachi, Toshiba, Yamaha, and Yasukawa who have supported the project.

\section{References}

[1] R. Clavel: DELTA4, a fast robot with parallel geometry, Proc. International Symposium on Industrial Robots, pp.92-100, 1988

[2] F. Pierrot, P. Dauchez and et al.: HEXA: A fast six-DOF fully- parallel robot, Proc. 5th International Conference on Advanced Robotics (Pisa), pp. 1158-1163, 1991

[3] S. Kawamura, W. Choe and et al.: Development of an Ultrahigh Speed Robot FALCON using Wire Drive System, Proc. IEEE International Conference on Robotics and Automation on (Nagoya), pp.215-220, 1995

[4] T. Arai, R. Stoughton and et al.: Development of a Parallel Link Manipulator, Proc. 5th International Conference on Advanced Robotics (Pisa), pp.839-844, 1991

[5] T. Arai, R Stoughton and et al.: Micro Hand Module Using Parallel Link Mechanism, Proc. Japan/USA Symposium on Flexible Automation (San Francisco), pp 163-168, 1992

[6] J. Hudgens and D. Tesar: A Fully-Parallel six Degree-ofFreedom Micro manipulator: Kinematic Analysis and Dynamic Model, Proc. 20th Biennial ASME Mechanisms Conference (Kissimmee), pp.29-37, 1988

[7] T. Arai, T. Tanikawa and et al.: Development of a New Parallel Manipulator with Fixed Linear Actuator, Proc. Japan/USA Symposium on Flexible Automation (Boston), Vol. 1, pp.145-149, 1996

[8] Y. Nakamura and M. Ghodoussi: Dynamics computation of closed-link robot mechanisms with non-redundant and redundant actuators, IEEE Trans. Robotics and Automation, Vol.5, No.3, pp.294-302, 1989

[9] T. Arai, R. Stoughton, et al.: Teleoperator Assisted Hybrid Control for Parallel Link Manipulator and Its Application to Assembly Task, Proc. 2nd International Symposium on Measurement and Control in Robotics (Tsukuba), pp.817-822, 1992

[10] M. P. Oliviers, J. R. Rene Mayer: Global Kinematic Calibration of a Stewart Platform, Proc. of ASME, DSC-Vol. 57-1, pp.129-136, 1995

[11] C. Wampler, J. Hollerbach, and T. Arai: An Implicit Loop Method for Kinematic Calibration and Its Application to Closed-Chain Mechanisms, IEEE Trans. Robotics \& Automation, Vol.11, No.5, pp.710-724

[12] J. M. Renders and et al.: Kinematic Calibration and Geometrical Parameter Identification for Robots, IEEE, trans. on Robotics and Automation, Vol.11, No. 5, pp. 770-724, 1995

[13] M. Goto and et al.: Calibration of coordinate system using laser trackers, Proc. $3^{\text {rd }}$ Annual Conf. Of ISMTII, Hayama, Japan, pp.59-65, 1996

[14]O. Nakamura and et al.: A laser tracking robot-performance calibration system using ball-seated bearing mechanisms and a spherically shaped cat's-eye retroreflector, Review of Scientific Instruments, Vol. 65, Part1 No. 4 pp. 1006-1011, 1994

[15] H. Nakamura: Industrial Robot Calibration Method and Its Application for Production Line, JRSJ, Vol. 15, No.2, pp. 16-20, 1997 (In Japanese) 